\title{
Quantitative single-cell characterization of CAR+ T cell effector functions
}

\author{
Ivan Liadi ${ }^{1}$, Harjeet Singh ${ }^{3}$, Gabrielle Romain ${ }^{1}$, Nicolas Rey-Villamizar², Amin Merouane ${ }^{2}$, Badrinath Roysam², \\ Laurence J Cooper $^{3}$, Navin Varadarajan ${ }^{1 *}$ \\ From Society for Immunotherapy of Cancer 28th Annual Meeting \\ National Harbor, MD, USA. 8-10 November 2013
}

Adoptive cell therapy (ACT) utilizing chimeric antigen receptor (CAR) T cells rendered specific for CD19 have demonstrated significant anti-tumor effects in patients with CD19+ chronic lymphocytic leukemia (CLL). In spite of the clinical promise of ACT in achieving complete responses, their efficacy remains unpredictable and new approaches are needed to address a priori define the therapeutic potential of $\mathrm{T}$-cell based therapies. In our current work, we characterize the in vitro functionality of CD19-specific (CD19RCD28) CAR+ T cells propagated using artificial antigen presenting cells expressing membrane bound IL-21, by employing a novel methodology single-cell nanowell screening that determines their cytotoxic ability and cytokine secretion capability at single-cell resolution. We show that CAR+ $\mathrm{T}$ cells exert specific cytotoxicity against NALM6 cells (31 $\pm 8 \%)$ when co-incubated at a 1:1 ratio in nanowell containers. Furthermore, single CAR $+\mathrm{T}$ cells were capable of engaging and killing multiple targets; $17 \pm 8 \%$ of $\mathrm{T}$ cells killed two target cells and $9 \pm 3 \%$ killed three target cells within the 6 hour window of observation. In parallel, microengraving was used to determine the cytokine secretion profile of these same cells. Hierarchical clustering of the two functions indicated that interferongamma (IFN $\gamma$ ) secretion is not correlated to cytotoxicity or the ability of T cells to kill multiple target cells. Simultaneously, monitoring apoptosis on CAR+ T cells allowed us to quantify their activation-induced cell death (AICD). $\mathrm{CAR}+\mathrm{T}$ cells that secreted IFN $\gamma$ upon target ligation did not undergo AICD whereas $\mathrm{T}$ cells that engaged in repeated killing showed an increased propensity to undergo AICD ( $\mathrm{p}=0.04)$. Dynamic time-lapse imaging of the interactions between CAR+ T cells and tumor cells indicated that the majority of CAR $+\mathrm{T}$ cells have high basal motility, form long-lived interactions with tumor cells $(50-100 \mathrm{~min})$ that lead to motility arrest and subsequent tumor-cell apoptosis. However, contact lifetimes or overall contact duration were not reliable predictors of subsequent tumor-cell apoptosis. Finally, kinetics of serial killing suggest that motile CAR+ T cells that form short-lived contacts exhibit rapid killing with very little motility arrest in vitro. In summary, our SNS based methodology allows the deep functional characterization of clinical grade CAR $+\mathrm{T}$ cells and can be used to: (1) determine in vitro functions of CAR+ T cells that correlate with clinical efficacy and (2) inform CAR design to maximize effector functionality while minimizing AICD.

\section{Authors' details}

'Chemical \& Biomolecular Engineering, University of Houston, Houston, TX, USA. ${ }^{2}$ Dept of Electrical Engineering, University of Houston, Houston, TX, USA. ${ }^{3}$ Pediatrics, University of Texas M.D. Anderson Cancer Center, Houston, TX, USA.

Published: 7 November 2013

\section{doi:10.1186/2051-1426-1-S1-P36}

Cite this article as: Liadi et al:: Quantitative single-cell characterization of CAR+ T cell effector functions. Journal for ImmunoTherapy of Cancer 2013 1(Suppl 1):P36. 